\title{
Twitter postings and suicide: An analysis of the postings of a fatal suicide in the 24 hours prior to death
}

\author{
By John F. Gunn and David Lester
}

Following the publication of Clues to Suicide by Edwin Shneidman and Norman Farberow (1957) which included one of the first studies of suicide notes, suicide notes have been frequently used to explore the psychodynamics of suicide. Shneidman (1981) argued that, when considered in the context of their life, suicide notes can provide insights into the meaning of the suicidal act of individuals. The study of suicide notes, however, is made difficult by the absence of a meaningful comparison group. Farberow and Shneidman (1961) compared genuine suicide notes with simulated notes written by non-suicidal people, but this may tell us only how well nonsuicidal individuals can fake a suicide note. Research has appeared comparing the genuine suicide notes left by men and by women and by those of different ages (e.g., Leenaars, 1988), overcoming this methodological problem.

More recently, the diaries left by occasioonal suicides have been studied, and these provide sequential information about the suicidal individuals, that is, how their state of mind changed as they got closer in time to their suicidal act. For example, Lester (2004) examined the diary of a young woman (Katie) who had died by suicide. One chapter of that book (Pennebaker \& Stone, 2004) examined

\section{ABSTRACT}

In the 24 hours prior to her death by suicide, a young girl made 145 posts on Twitter. In the posts, she outlines her history of incestuous sexual abuse, rape, and risky sexual behavior. These posts were analyzed using the Linguistic Inquiry and Word Count (LIWC). Trends were found indicating an increase in positive emotions over the 24 hours and a change in focus from the self to others.

This first study of posts on Twitter by a person about to commit suicide provides hypotheses for future studies of this medium for communication. the diary using the Linguistic Inquiry and Word Count (LIWC) developed by Pennebaker, Francis, and Booth (2001). They found that Katie's writings showed an increase in positive and a decrease in negative emotions closer to her death. Similarly Barnes, Lawal-Solarin, and Lester (2007) examined the letters written by a young man who committed suicide, and found that over time the overall positive nature of the letters increased. Lester (2009) examined the diary of Cesare Pavese, also using the LIWC, and found the same trend as Pennebaker, et al. (2001) had found with Katie's diary - an increase in positive emotions and a decrease in negative emotions over time.

Today, with the advent of modern means for communication, suicidal individuals are using the Internet to post suicidal communications including suicide notes and videos. Facebook, Youtube and Twitter have become popular for such communications. These differ from suicide notes in that suicide notes are meant to be read after the individual has committed suicide, whereas postings on the Internet are meant to be read before the individual dies, and in occasional cases those reading or viewing the postings have alerted authorities and prevented the potential suicide from engaging in lethal actions.
Although the diaries of suicidal individuals permit the study of the suicidal mind over time, the writings span months and even years. The present study examines postings made on a social networking website by a young girl in the final 24 hours prior to her death. This permits the study of the suicidal mind in the hours prior to the suicidal act. In reports based on the hospital notes made by staff of psychiatric patients who committed suicide while hospitalized, it has been noted that the mood of the suicides improved in the hours prior to their suicidal act (Clements, Bonacci, Yerevanian, Privitera \& Kiehne, 1985; KeithSpiegel \& Spiegel, 1967). The present study offers a unique contribution to this field of study, therefore, by analyzing the writings left by a suicide for changes occurring in the final hours and minutes prior to a fatal act. No study has yet examined the written texts left by a suicide in this way.

\section{Methods}

For the purposes of anonymity, the young girl will be referred to as Abby, although information on her is available on the Internet*. After Abby's suicide, her twitter account was closed, making retrieval of the original posts impossible.

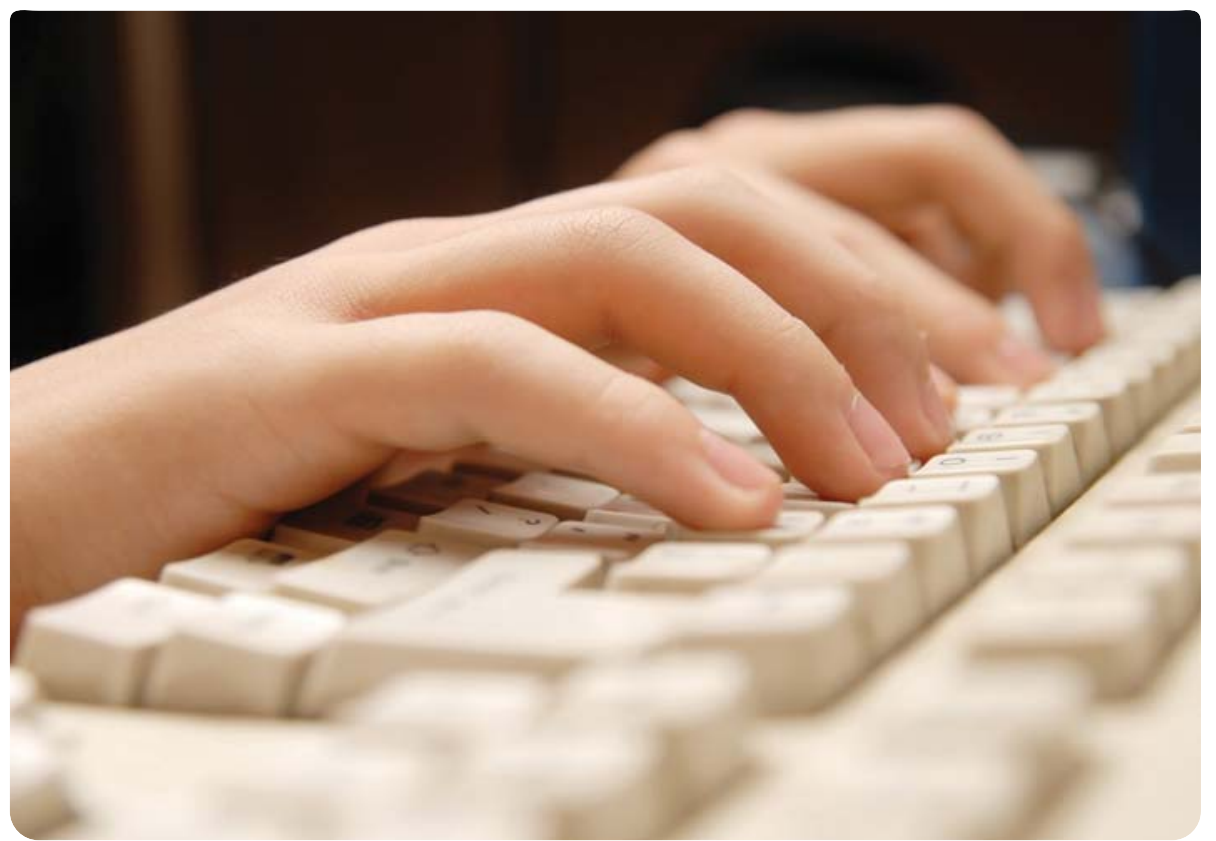

*http://www.myfoxhouston.com/story/18192240/18-year-old-girl-tweets-144-times-before-committing-suicide 
Table 1.

Correlations over postings on Twitter. Only statistically significant correlations are shown.

However, using an Internet search engine, the twitter posts were found reproduced in other websites and downloaded for the present analysis. News articles related to the suicide were found to contain quotes from the twitter postings, which matched the reproduced twitter postings, leading to the conclusion that the twitter postings downloaded were accurate.

These twitter postings were analyzed using the LIWC developed by Pennebaker, Francis, and Booth (2001). This program analyzes texts word-by-word. The program can count pronouns (e.g., "I", "Me"), emotional words (e.g., negative and positive), and particular words (e.g., "death"). For most of the categories, the scores are expressed as percentages of the total text.

The first analysis analyzed all 145 twitter postings, labeled from 1 to 145 . For the second and third analyses, since Twitter postings are brief (limited to 140 characters), the postings were combined. Postings made within seconds of each other (for example, three postings made at 9:45 pm, two postings made at 10:10 $\mathrm{pm}$, and so on) were combined into single postings and, next, postings made within the same hour (for example, all posts from 10:00 pm-10:59 pm) were combined for two separate analyses.

\section{Results}

The Pearson correlations are shown in Table 1.

\section{Individual Posts}

In the first analysis, the 145 posts were analyzed separately. As the postings got closer to Abby's death, they increased in length and in the number of words per sentence (Pearson $r=.547$, $\mathrm{p}<.001$, $r=.324, \mathrm{p}<.001$, respectively). There was also a decrease in references to the present and, although only trending toward significance, an increase in the references to the past $(r=-.267, \mathrm{p}<$ $.001, r=.155, \mathrm{p}<.10$, respectively).

\section{Posts by Minute}

In the second analysis, postings made within the same minute (for example, all postings made at 9:45) were combined into single texts, resulting in 75 texts to be compared.

\begin{tabular}{|c|c|c|c|c|}
\hline & \multicolumn{3}{|c|}{ Correlations over postings } & \multirow[b]{3}{*}{ Examples of words } \\
\hline & $\begin{array}{l}\text { Individual } \\
\text { postings } \\
n=145\end{array}$ & $\begin{array}{l}\text { Postings } \\
\text { combined } \\
\text { by minute } \\
n=75\end{array}$ & $\begin{array}{l}\text { Postings } \\
\text { combined } \\
\text { by hour } \\
n=6\end{array}$ & \\
\hline & $r$ & $r$ & $\mathbf{r}$ & \\
\hline Word count & $.55^{\star * *}$ & $.23 *$ & .24 & \\
\hline Words per sentence & $.32 * * *$ & $-.28 *$ & -.11 & \\
\hline Unique words & $-.38 * * *$ & -.18 & -.27 & \\
\hline Words with $6+$ letters & -.04 & -.04 & $-.90 *$ & \\
\hline Pronoun & -.08 & $-.24^{*}$ & -.64 & I, they \\
\hline I & .01 & -.08 & $-.81 \neq$ & I, me \\
\hline Self & -.02 & -.10 & $-.85^{*}$ & we, me \\
\hline Negations & -.03 & $.19 \neq$ & .39 & no, never \\
\hline Assents & $-.20 *$ & $-.26 *$ & $-.82 *$ & yes, ok \\
\hline Articles & $.21^{*}$ & $.24^{*}$ & .40 & $a$, the \\
\hline Prepositions & $.20 *$ & .19 & -.36 & on, to \\
\hline Positive emotions & -.11 & -.06 & $.75 \ddagger$ & happy, good \\
\hline Anger & $-.14 \neq$ & $-.20 \neq$ & -.66 & hate, pissed \\
\hline Cognitive processes & -.13 & -.12 & $.73 \neq$ & cause, know \\
\hline Discrepancies & .01 & .05 & $.79 \neq$ & should, could \\
\hline Tentative & -.10 & -.08 & $.78 \neq$ & maybe, perhaps \\
\hline Friends & .02 & $.21 \neq$ & .37 & buddy, coworker \\
\hline Family & $.15 \neq$ & .18 & .35 & brother, cousin \\
\hline Past tense & $.16 \neq$ & .08 & .31 & \\
\hline Present tense & $-.27 * * *$ & -.16 & .42 & \\
\hline Space & $-.14 \neq$ & $-.12 \ddagger$ & -.64 & over, up \\
\hline Inclusive & .01 & .01 & $.81 \neq$ & with, include \\
\hline School & $.17 *$ & .13 & .45 & class, student \\
\hline Physical states & -.08 & $-.23 \neq$ & -.64 & ache, breast \\
\hline Sleep & $-.22 * *$ & $-.26 *$ & -.10 & asleep, bed \\
\hline Swearing & $-.14 \neq$ & $-.20 \neq$ & -.66 & \\
\hline
\end{tabular}

As with the first analysis, there was an increase in word count but, in this analysis, there was a decrease in words per sentence $(r=.234, \mathrm{p}<.05, r=$ $.283, \mathrm{p}<.05$, respectively). There was a decrease in the use of anger words, though this was only trending towards significance $(r=-.199, \mathrm{p}<.10)$. Also trending towards significance, was an increase in references to friends $(r=.210, \mathrm{p}<.10)$.

\section{Posts by Hour}

For the final analysis, postings made within the same hour (for example, all posts made between 10:00 am and 10:59 am) were combined. Though only trending towards significance, there was a decrease in the use of the pronoun "I" $(r=-.805, \mathrm{p}<.10)$. There was also a significant decrease in references to the self $(r=-.852, p<.05)$. There was an increase in positive emotion terms, although this too was only trending towards signifi-cance $(r=.745, \mathrm{p}<.10)$ and non-signi-ficant decrease in negative emotions.

The only finding that was statistically significant for all three analyses was that the use of assents (such as "yes" "ok" "mhmm") decreased over time $(r=$ $.202, \mathrm{p}<.05, r=-.256, \mathrm{p}<.05, r=$ $.819, \mathrm{p}<.05$, respectively). 


\section{Discussion}

Several findings support the findings of previous research into written materials left behind by suicides. As with Lester (2004) and Lester (2009), there was an increase in positive emotions in the posts closer to death, but this finding was only trending towards significance. This could be a result of the brevity of each post. When examined individually, and even by the minute, each post was relatively short, while when compared by the hour, there was more depth to the writings. Also of interest were the decreases in the use of "I" and the decrease in references to the self, though the decrease in the use of "I" was only trending toward significance. Pennebaker and Stone (2004) found a similar trend over time in Katie's diary, and Lester (2010) found a similar change in two tape recordings made by an 18-year old adolescent in the hours before he shot himself. This young man was very self-oriented in a tape recording made six hours prior to his suicide, but much more otheroriented in the tape recording made two hours prior to his suicide.

The increase in positive emotions in the third analysis and the decrease in negative emotions, although they did not reach statistical significance using two-tailed statistical tests, are consistent with the earlier studies by Lester on Katie's diary and on the letters of a young man. More importantly, they do confirm clinical reports on patients residing on psychiatric wards who have been found, in retrospect, to show an improvement in mood in the hours prior to their suicide in the hospital (Clements, et al., 1985; Keith-Spiegel \& Spiegel, 1967).

There were several limitations to the present study. The first limitation involves the authenticity of the tweets. Though we have a reasonable expectation to believe the postings are authentic (news reports quoted them), because they were obtained from a secondary source and not the original website, there is some marginal doubt as to their authenticity. A second limitation of the study involves the nature of the postings. Each posting was relatively short and, when combined to be of an acceptable length (in the third

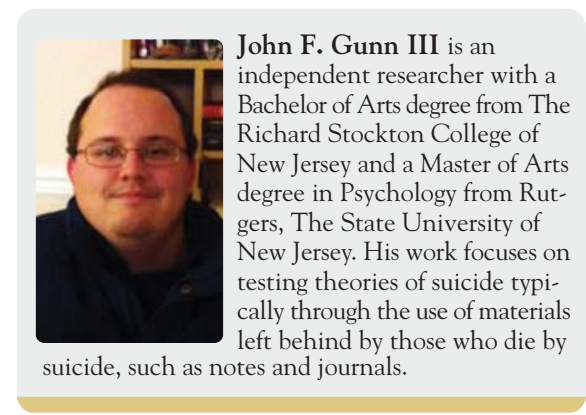

(a)

David Lester has doctoral degrees from Cambridge University (UK) in social and political science and Brandeis University (USA) in psychology. He is Distinguished Professor of Psyc hology at the Richard Stockton College of New Jersey. Lester has published extensively on suicide, murder and other issues in thanatology. His recent book include Katie's Diary: Unlocking the Mystery of a Suicide (2004), Suicide and the Holocaust (2005), Is there Life after Death? (2005) and Understanding Suicide: Closing the Exits Revisited (2009).

analysis), there were only six postings for comparison. Future research should make use of longer postings, if available. Finally, upon reading the posts, it becomes clear that Abby attempted to write the posts using rhyming. For example: 10:21 am I went to the bathroom and locked the door; 10.20 am I took apart a razor I had gotten from the store. As a result of her attempt to write poetically, Abby may have used certain words that would not have been used if writing a suicide note or a journal entry. However, not all of the tweets rhymed in this way, and we feel that this was only a minor limitation.

One problem with using the LIWC program is that it assesses 74 variables, and so there should be a correction for multiple tests which typically (and in the present case) eliminates all of the statistically significant results. The results of the present study should, therefore, be viewed as providing specific hypotheses for future studies on posts and notes left by suicides which, if confirmed, would eliminate the problem of multiple statistical tests.

This study makes use of a new form of writing left behind by suicides, made possible by the rising use of technology and social networking sites. It opens the door to further research using online postings in the hours and minutes prior to death by suicide. For example, Lester (2010) analyzed two tape recordings left by a young man in the hours prior to his suicide. Future research may be able to use e-mails made during crises, telephone conversations, video recordings, and text messages. This research depends in some cases on the ability to save materials available over the Internet and, in other cases, the generosity of those who lose a significant other to suicide sharing material left behind by the deceased, as in the case of the tape recordings analyzed by Lester (2010).

This research is important because it permits to study the mind of suicides as they approach their act. It allows us to monitor short-term indications of future suicidal behavior (that is, warning signs) rather than the long-term predictors for suicide (risk factors).

\section{References}

Barnes, D. H., Lawal-Solarin, F. W., \& Lester, D. (2007). Letters from a suicide. Death Studies, 31, 671-678.

Clements, C., Bonnaci, D., Yerevanian, B., Privitera, M., \& Kiehne, L. (1985). Assessment of suicide risk in patients with personality disorder and major affective disorder. Quality Review Bulletin, 11, $150-154$.

Farberow, N. L., \& Shneidman, E. S. (1961). The cry for help. New York: McGraw-Hill.

Keith-Spiegel, P., \& Spiegel, D. E. (1967). Affective states of patients immediately preceding suicide. Journal of Psychiatric Research, 5, 89-93.

Leenaars, A. A. (1988). Suicide notes. New York: Human Science Press.

Lester, D. (Ed). (2004). Katie's Diary. New York: Brunner-Routledge.

Lester, D. (2009). Learning about suicide from the diary of Cesare Pavese. Crisis: The Journal of Crisis Intervention and Suicide Prevention, 30, 222-224. Lester, D. (2010). The final hours. Psychological Reports, 106, 791-797.

Pennebaker, J. W., Francis, M. E., \& Booth, R. J. (2001). Linguistic inquiry and word count. Mahwah, NJ: Erlbaum.

Pennebaker, J. W., \& Stone, L. D. (2004). What was she trying to say. In D. Lester (Ed.), Katie's diary (pp. 55-79). New York: Brunner-Routledge.

Shneidman, E. S. (1981). Suicide notes and tragic lives. Suicide \& Life-Threatening Behavior, 11, 286-299.

Shneidman, E. S., \& Farberow, N. L. (1957). Clues to suicide. New York: McGraw-Hill. Stirman, S. W. \& Pennebaker, J. W. (2001). Word use in the poetry of suicidal and non-suicidal poets. Psychosomatic Medicine, 63, 517-522.

Stirman, S. W., \& Pennebaker, J. W. (2001). Word use in the poetry of suicidal and non-suicidal poets. Psychosomatic Medicine, 63, 517-522. 